\title{
Consumo de alimentos protetores e preditores do risco cardiovascular em adultos do estado de Pernambuco ${ }^{1}$
}

\author{
Consumption of foods that protect against \\ and promote cardiovascular disease by \\ adults from Pernambuco State, Brazil
}

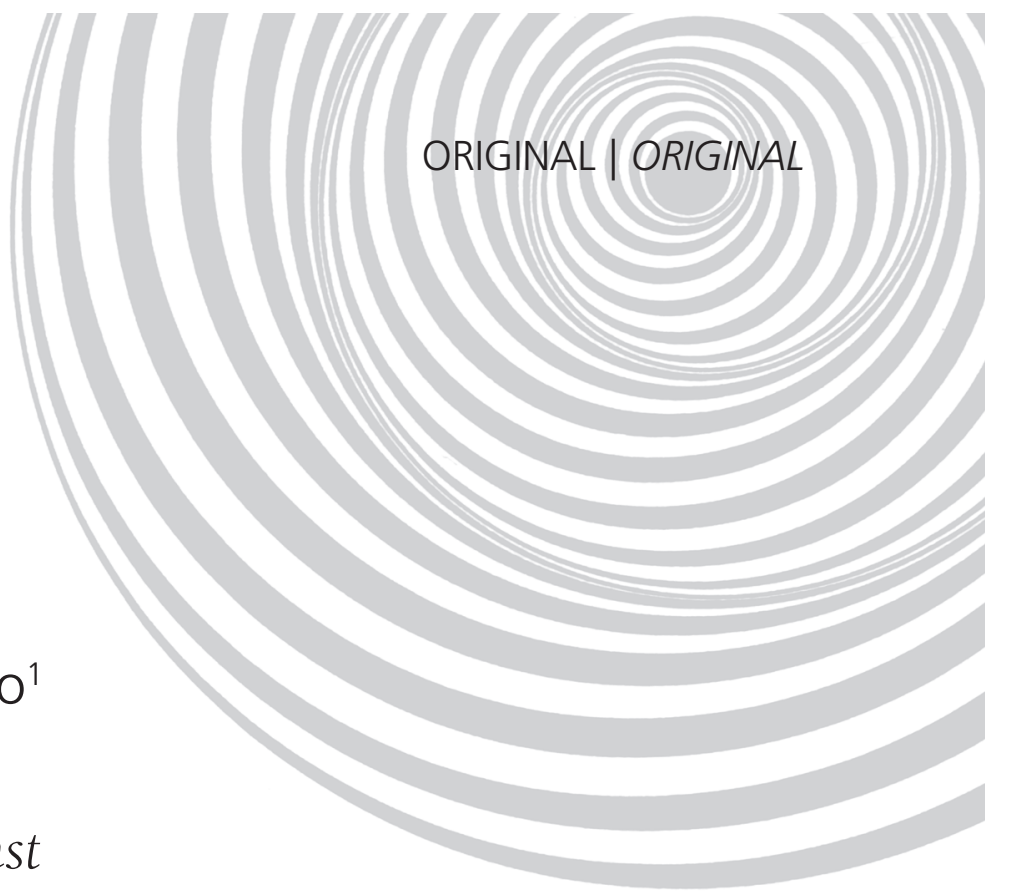

Cláudia Porto Sabino PINHO²

Alcides da Silva DINIZ²

IIma Kruze Grande de ARRUDA²

Pedro Israel Cabral de LIRA ${ }^{2}$

Poliana Coelho CABRAL ${ }^{2}$

Leopoldina Augusta Souza SIQUEIRA²

Malaquias BATISTA FILHO

RE S U M O

\section{Objetivo}

Avaliar o consumo de alimentos protetores e preditores do risco cardiovascular e os fatores associados na população adulta do Estado de Pernambuco.

\section{Métodos}

Estudo transversal, de base populacional, envolvendo 1820 adultos de 25 a 59 anos. Foram constituídos 3 grupos de alimentos: fontes em fibras (protetores), em carboidratos simples e em gorduras saturadas (risco para doenças cardiovasculares e ganho excessivo de peso). O consumo alimentar foi avaliado por um questionário de frequência alimentar com mensuração convertida em escores. O modelo conceitual considerou variáveis socioeconômicas, demográficas, comportamentais e antropométricas.

\section{Resultados}

O escore médio do consumo de alimentos-fonte em carboidratos simples foi maior que o consumo de alimentos-fonte de fibras e gorduras saturadas $(p<0,001)$. Um menor consumo do grupo de alimentos de risco foi

\footnotetext{
1 Artigo elaborado a partir da dissertação de CPS PINHO, intitulada "Excesso de peso e distribuição corporal de gordura: magnitude e fatores associados em adultos do Estado de Pernambuco". Universidade Federal de Pernambuco; 2011. Apoio: Conselho Nacional de Desenvolvimento Científico e Tecnológico (CNPq), (Processos No 505540/2004-5 e 501989/2005-4).

2 Universidade Federal de Pernambuco, Centro de Ciências da Saúde, Departamento de Nutrição. Av. Prof. Moraes Rego, 1235, Cidade Universitária, 50670-901, Recife, PE, Brasil. Correspondência para/Correspondence to: CPS PINHO. E-mail: <claudiasabinopinho@hotmail.com>.

3 Instituto de Medicina Integral Professor Fernando Figueira, Diretoria de Pesquisa. Recife, PE, Brasil.
} 
342 CPS PINHO et al.

observado em indivíduos de maior idade, provenientes de área rural, com menor renda e escolaridade. Maior consumo de carboidratos simples foi identificado entre indivíduos com baixo peso, e o consumo mais elevado de gorduras saturadas foi verificado em indivíduos não fumantes e naqueles que relataram o consumo de álcool. O consumo de fibras foi superior em indivíduos não fumantes e naqueles de maior renda e escolaridade.

\section{Conclusão}

O maior consumo de carboidratos simples, em detrimento do consumo de fibras, está relacionado à suscetibilidade do indivíduo a doenças provocadas pela má alimentação e configura o processo de transição nutricional experimentado pelo Brasil nas últimas décadas.

Termos de indexação: Carboidratos. Consumo de alimentos. Doenças cardiovasculares. Gorduras.

\section{A B S T R A C T}

\section{Objective}

The present study assessed the consumption of foods that protect against and promote cardiovascular diseases and associated factors by adults from Pernambuco.

\section{Methods}

This cross-sectional, population-based study done in 2006 involved 1820 adults aged 25 to 59 years. Foods were divided into three groups: high-fiber (protective foods), sugary foods and high-saturated fat foods (risk of cardiovascular disease and excess weight gain). Food intake was assessed by a food frequency questionnaire and amounts were converted into scores. The model included demographic, socioeconomic, behavioral and anthropometric variables.

\section{Results}

The mean sugary food intake score was higher than the high-fiber and high-saturated fat intake scores $(p<0.001)$. Older country people with lower income and education level consumed less sugary foods and saturated fats. The highest high-fiber food scores were associated with higher income and education level. Low-birth weight individuals presented a high intake of simple carbohydrates; nonsmokers and those who reported consuming alcoholic beverages presented a high intake of saturated fats. Fiber intake was higher among nonsmokers and individuals with higher income and education level.

\section{Conclusion}

Higher intake of sugary foods instead of high-fiber foods makes people more susceptible to diet-related diseases and reflects the nutritional transition that has been going on in Brazil in the last decades.

Indexing terms: Carbohydrates. Food consumption. Cardiovascular diseases. Fats.

\section{N T R O D U ÇÃ O}

Estudos epidemiológicos têm fornecido evidências de que dietas inadequadas e muitas vezes autoprescritas potencializam os riscos para Doenças Cardiovasculares (DCV), diabetes Mellitus, neoplasias e outras doenças crônicas não-transmissíveis $^{1,2}$.

O padrão de consumo alimentar da população brasileira vem sofrendo significativas modificações durante as últimas décadas, como consequência direta de mudanças estruturais que vêm ocorrendo no país ${ }^{3}$. O aumento do consumo de alimentos com alto teor de carboidratos simples ou lipídeos tem gerado significativa elevação da densidade calórica do padrão alimentar populacional $^{4,5}$, e, associado ao consumo inadequado de frutas, vegetais e fibras, é um dos elementos que configuram o processo de transição nutricional, primariamente derivado de variações na renda da população, apelos mercadológicos, preços relativos dos alimentos, grau de urbanização, estrutura de oferta alimentar, nível educacional e influências culturais diversas ${ }^{6}$.

Os dados sobre consumo alimentar, juntamente com outros indicadores do estado nutricional, segurança alimentar, morbidade e risco de doenças, são as bases para o monitoramento das 
tendências dietéticas e a definição de políticas para agricultura, economia e saúde ${ }^{7}$.

O consumo alimentar inadequado, principalmente quando associado ao sedentarismo, desponta entre os problemas mais prevalentes e de maior risco populacional. Os hábitos alimentares apresentam-se como marcadores de risco para doenças cardiovasculares na medida em que o consumo elevado de colesterol, de lipídeos e de ácidos graxos saturados, somado ao baixo consumo de fibras, participa na etiologia das dislipidemias, obesidade, diabetes e hipertensão $0^{8,9}$.

Há na literatura diversos estudos no país que enfocam os determinantes do consumo de frutas, legumes e hortaliças ${ }^{10-12}$, mas são escassas as investigações que avaliam os fatores associados ao consumo de alimentos-fonte em carboidratos simples e gorduras saturadas, componentes reconhecidamente associados ao ganho excessivo de peso e às DCV. Além disso, ainda são escassos os estudos do consumo alimentar na população da região Nordeste, sobretudo provenientes de amostras representativas e que considerem a população estadual.

Este estudo objetiva avaliar o consumo de alimentos protetores e preditores do risco cardiovascular e os fatores associados na população adulta do estado de Pernambuco.

\section{M É T O D O S}

Foi realizado um estudo de corte transversal, de base populacional, envolvendo adultos na faixa etária de 25 a 59 anos, de ambos os sexos, provenientes de área rural e urbana do Estado de Pernambuco, no período de maio a outubro de 2006.

Na definição do tamanho amostral, foi considerada uma frequência do consumo de frutas e hortaliças em homens recifenses estimada em $17,1 \%{ }^{13}$, um intervalo de confiança de $95,0 \%$, precisão de 3,0\% e um fator de correção amostral de 2,5\%, perfazendo um total mínimo de 1512 indivíduos. Para compensar eventuais perdas e permitir um melhor nível de estratificação das variáveis independentes, esse tamanho amostral foi aumentado em $20,0 \%$, resultando em uma amostra final de 1820 indivíduos.

O processo de seleção da amostra (probabilística e estratificada) desenvolveu-se em 3 estágios: 1) sorteio dos municípios, 2) sorteio dos setores censitários (unidades territoriais demarcadas pelo Instituto Brasileiro de Geografia e Estatística - IBGE) e 3) sorteio aleatório dos domicílios dentro de cada setor censitário para selecionar as famílias e os adultos elegíveis para o estudo. Foi estudado o número máximo de 40 $( \pm 5)$ unidades amostrais por setor censitário.

A partir do sorteio aleatório, foram selecionados 18 municípios e considerados três estratos geográficos: Região Metropolitana do Recife (RMR), Interior Urbano (IU) e Interior Rural (IR). Para definição da quantidade de setores censitários a serem estudados, foi considerado o número com representações proporcionais à população dos municípios selecionados, figurando 16 setores censitários da RMR, 17 do IU e 12 do IR.

Os dados referentes ao consumo alimentar foram coletados por meio de um Questionário de Frequência Alimentar (QFA) que possibilitou ao entrevistado informar o número de vezes que consumiu determinado alimento em quatro categorias de frequência: consumo diário, consumo semanal, consumo mensal e raramente/nunca consome. Para este estudo foram avaliados 15 alimentos ou preparações, e, para que a frequência de consumo de cada item fosse tratada como variável quantitativa, foi atribuído um peso (S) a cada categoria de frequência. O modelo proposto por Fornés et al. ${ }^{14}$, com adaptações, foi utilizado na transformação dessas diferentes categorias em frequência em consumo mensal. Foi definido um valor de peso máximo - $\mathrm{S}=1$ - para os alimentos com consumo diário, e os demais pesos das categorias de frequência foram obtidos de acordo com a seguinte equação: $S=(1 / 30) \times n$, sendo $n$ o número de dias que o indivíduo avaliado consumiu determinado alimento. 
Após o cálculo do peso da frequência de consumo de cada item, os alimentos analisados foram inseridos em três grupos: o grupo I foi composto por alimentos-fonte de fibras (leguminosas, frutas, legumes e hortaliças); o grupo II foi composto pelos alimentos-fonte de carboidratos simples (bolo, biscoito, açúcar e refrigerantes); e no grupo III foram inseridos os alimentos-fonte de gorduras saturadas (carnes com gordura, frango com pele, vísceras, embutidos, laticínios, gorduras e frituras). O grupo I foi considerado protetor para as DCV e para o ganho excessivo de peso, e os grupos II e III foram compostos por alimentos considerados de risco ou preditores para o ganho excessivo de peso e para o desenvolvimento de $D C V$, respectivamente. Posteriormente, foi calculado o escore de frequência do grupo através do somatório do peso de cada item.

O modelo conceitual para explicar o consumo alimentar considerou variáveis socioeconômicas, demográficas, comportamentais e antropométricas. Dentre as variáveis socioeconômicas e demográficas, consideraram-se: sexo, idade (categorizadas nos intervalos $25 \vdash 30$ anos; $30 \vdash 40$ anos; $40 \vdash 50$ anos e 50-60 anos), área geográfica da residência (Região Metropolitana do Recife, Interior Urbano, Interior Rural); escolaridade em anos de estudo (categorizada em 0-4 anos, 5-8 anos e $\geq 9$ ) e renda familiar em salários-mínimo per capita (estabelecida em quartis de renda).

Dentre as variáveis comportamentais, avaliaram-se o tabagismo, o consumo de álcool e o nível de atividade física. Para o tabagismo, foram consideradas as categorias: fumante (o indivíduo que referiu o hábito de fumar), não fumante (o indivíduo que relatou nunca haver fumado) e ex-fumante (o indivíduo que referiu o hábito de fumar em algum momento da vida, mas que não o fazia na ocasião da aplicação do questionário). Para a variável consumo de álcool, avaliou-se o consumo de bebidas alcoólicas nos 30 dias anteriores à aplicação do questionário, sendo considerada a resposta dicotômica sim ou não.

Para determinação do nível de atividade física, foi utilizado o International Physical Activity
Questionnaire (IPAQ) ${ }^{15}$, 2001, em sua versão curta, que leva em consideração as quatro dimensões da atividade física: no lazer, atividades domésticas, atividades ocupacionais e atividades relacionadas ao deslocamento. Esse instrumento mede a frequência e a duração das atividades físicas moderadas, vigorosas e caminhadas realizadas na última semana por pelo menos dez minutos contínuos, incluindo exercícios, esportes, atividades físicas ocupacionais e de recreação realizadas em casa, no tempo livre, como meio de transporte e no lazer.

Foi construído um escore de atividade física em minutos por semana, somando-se os minutos despendidos em caminhada e atividades de intensidade moderada aos minutos despendidos em atividades de intensidade vigorosa multiplicados por dois. Essa estratégia visa considerar as diferentes intensidades de cada atividade, e está de acordo com as recomendações atuais quanto à prática de atividade física ${ }^{16}$. Um escore abaixo de 150 minutos por semana foi o ponto de corte utilizado para classificar os indivíduos como insuficientemente ativos ou sedentários.

Para as variáveis antropométricas, foram considerados o Índice de Massa Corporal (IMC), segundo os pontos de corte preconizados pela Organização Mundial de Saúde (OMS) ${ }^{17}$, e a Circunferência da Cintura (CC), que foi categorizada segundo o sexo, adotando-se o ponto de corte da $\mathrm{OMS}^{17}$, que considera risco cardiovascular elevado quando $C C>80 \mathrm{~cm}$ para mulheres $\mathrm{e}$ CC $>94 \mathrm{~cm}$ para homens.

A coleta das medidas antropométricas foi realizada por duplas de técnicos treinados em antropometria. A altura foi determinada com estadiômetro portátil - (Alturaexata Ltda ${ }^{\circledR}$ ) -, milimetrado, com precisão de até $(1 \mathrm{~mm})$ em toda sua extensão. Os indivíduos foram colocados em posição ereta, descalços, com membros superiores pendentes ao longo do corpo, os calcanhares, o dorso e a cabeça tocando a coluna de madeira. O peso dos adultos foi obtido por meio de balança digital (Modelo MEA-03200/Plenna ${ }^{\circledR}$ ), com capacidade de $150 \mathrm{~kg}$ e escala de 100 gramas, com o indivíduo descalço e indumentária mínima. 
Para garantir a acurácia das mensurações, foram aferidas duas medidas de peso, altura e CC. Quando a diferença entre as avaliações excedia $0,5 \mathrm{~cm}$ para altura e CC e $100 \mathrm{~g}$ para o peso, repetia-se a mensuração e anotavam-se as duas medições com valores mais próximos, sendo utilizada sua média para registro.

Os indivíduos que concordaram em participar da pesquisa assinaram o termo de Consentimento Livre e Esclarecido. O protocolo de estudo foi submetido à apreciação do Comitê de Ética em Pesquisa em Seres Humanos do Instituto de Medicina Integral Professor Fernando Figueira (IMIP).

Os escores de frequência de consumo alimentar, em virtude de se tratar de uma mensuração em escala ordinal, foram descritos sob a forma de mediana e Intervalo Interquartílico (IQ). A associação entre consumo alimentar e variáveis explicativas foi avaliada pelos testes " $U$ " de Mann Whitney (duas medianas) e Kruskal Wallis (mais de duas medianas), empregando-se o teste " $U$ " de Mann Whitney a posteriori. Na validação das associações investigadas foi adotado o valor de $p<0,05$.

A base de dados foi compilada no Programa Epi Info versão 6.04 (CDCNHO, Atlanta, GE, USA), com dupla entrada, e posterior uso do módulo validate para checar eventuais erros de digitação. Para as análises estatísticas, foi empregado o pacote estatístico Statistical Package for the Social Sciences (SPSS) versão 12.0 (SPSS Inc., (hicago, IL, USA).

\section{RES ULTADOS}

Dos 1820 indivíduos avaliados, 2,2\% foram excluídos por serem gestantes, e houve $11,0 \%$ de perdas (ausência de respostas e inconsistências de informações), totalizando 1580 adultos efetivamente estudados.

O perfil da população avaliada mostrou uma mediana de idade de 33 anos $(I Q=29-42)$, $58,0 \%$ de mulheres, $48,7 \%$ residentes no Interior
Rural (IR) do Estado e 54,3\% com baixa escolaridade ( $<5$ anos de estudo) (Tabela 1).

Tabela 1. Características demográficas, socioeconômicas, comportamentais e antropométricas de adultos do Estado de Pernambuco, 2006.

\begin{tabular}{|c|c|c|c|}
\hline \multirow{2}{*}{ Variáveis } & \multirow[b]{2}{*}{$\mathrm{N}$} & \multicolumn{2}{|c|}{ Total } \\
\hline & & $\mathrm{n}$ & $\%$ \\
\hline Sexo & 1580 & & \\
\hline Masculino & & 664 & 42,0 \\
\hline Feminino & & 916 & 58,0 \\
\hline Idade (anos) & 1580 & & \\
\hline $25 \mid-30$ & & 485 & 30,7 \\
\hline $30 \mid-40$ & & 633 & 40,1 \\
\hline $40 \mid-50$ & & 290 & 18,4 \\
\hline $50 \mid-60$ & & 172 & 10,9 \\
\hline Área de residência & 1580 & & \\
\hline Região metropolitana do Recife & & 422 & 26,7 \\
\hline Interior urbano & & 389 & 24,6 \\
\hline Interior rural & & 769 & 48,7 \\
\hline Escolaridade (anos de estudo) & 1563 & & \\
\hline $0 \mathrm{H} 4$ & & 849 & 54,3 \\
\hline $5 \mid-18$ & & 349 & 22,3 \\
\hline$\geq 9$ & & 365 & 23,1 \\
\hline Renda familiar per capita (salários-mínimo) & 1560 & & \\
\hline$\leq 0,12$ & & 393 & 24,9 \\
\hline $0,12-0,25$ & & 412 & 26,1 \\
\hline $0,25-0,45$ & & 367 & 23,2 \\
\hline$\geq 0,45$ & & 388 & 24,6 \\
\hline Tabagismo & 1579 & & \\
\hline Fumante & & 353 & 22,4 \\
\hline Não fumante & & 987 & 62,5 \\
\hline Ex-fumante & & 239 & 15,1 \\
\hline Atividade física* & 1580 & & \\
\hline Suficientemente ativos & & 1130 & 71,5 \\
\hline Insuficientemente ativos & & 450 & 28,5 \\
\hline Consumo de álcool nos últimos 30 dias & 1476 & & \\
\hline Não & & 1017 & 68,9 \\
\hline $\operatorname{sim}$ & & 459 & 31,1 \\
\hline Estado nutricional $^{+}$ & 1580 & & \\
\hline Baixo Peso $\left(\mathrm{IMC}<18,5 \mathrm{~kg} / \mathrm{m}^{2}\right)$ & & 42 & 2,7 \\
\hline Eutrofia (IMC $\geq 18,5 \mathrm{~kg} / \mathrm{m}^{2}$ e $\left.<25 \mathrm{~kg} / \mathrm{m}^{2}\right)$ & & 731 & 46,3 \\
\hline Excesso de Peso $\left(\mathrm{IMC} \geq 25 \mathrm{~kg} / \mathrm{m}^{2}\right)$ & & 807 & 51,1 \\
\hline Circunferência da cintura & 1580 & & \\
\hline Normal & & 769 & 48,1 \\
\hline Elevada ${ }^{\ddagger}$ & & 820 & 51,9 \\
\hline $\begin{array}{l}\text { *Suficientemente ativos: atividades moderada, vi } \\
\text { por tempo } \geq 150 \text { minutos/semana. Insuficientem } \\
\text { com atividades moderada, vigorosa ou caminha } \\
{ }^{\dagger} \text { OMS, } 1998 .{ }^{\ddagger} \text { Circunferência da Cintura Elevada: } \\
\text { e }>94 \mathrm{~cm} \text { para homens. }\end{array}$ & $\begin{array}{l}\text { igorosa } \\
\text { nente ati } \\
\text { ada }<15 \\
:>80 \mathrm{~cm}\end{array}$ & $\begin{array}{l}\text { ou cam } \\
\text { ivos: ind } \\
\text { Omin/s } \\
\text { para m }\end{array}$ & $\begin{array}{l}\text { inhada } \\
\text { divíduos } \\
\text { emana } \\
\text { ulheres }\end{array}$ \\
\hline
\end{tabular}


O escore médio do consumo de alimentos-fonte de carboidratos simples (Média - $M=0,87$, Desvio-Padrão - $\mathrm{DP}=0,42)$ foi superior ao consumo de alimentos-fonte de fibras $(M=0,51, D P=0,23)$ e aproximadamente três vezes maior que o consumo de alimentos-fonte de gorduras saturadas $(\mathrm{M}=0,30, \mathrm{DP}=0,13)(p<0,001)$ (Figura 1).

Houve um menor consumo de alimentos-fonte de carboidratos simples e gorduras saturadas entre os indivíduos de maior idade. Verificou-se similaridade das medianas dos escores de consumo dos três grupos na distribuição entre os sexos (Tabela 2).

Um menor consumo de alimentos dos três grupos foi evidenciado em indivíduos de menor escolaridade (0-4 anos de estudo).

Concernente à distribuição do espaço geográfico, observou-se que o consumo de alimentos protetores (Grupo I) foi similar entre os indivíduos de todos os estratos geográficos considerados. Em relação aos outros grupos de alimentos, os resultados apontaram menores medianas de escores de consumo no IR.

Observou-se que o escore de consumo dos três grupos de alimentos aumentou pari passu com a progressão da renda. Maiores medianas

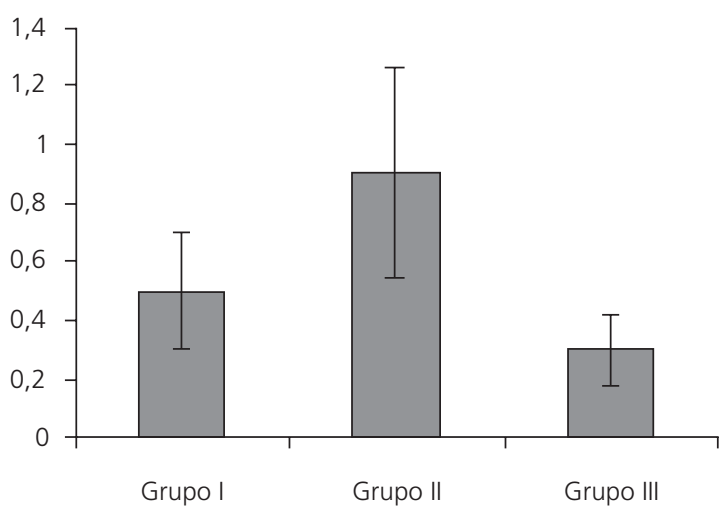

Figura 1. Escore (média e desvio-padrão) do consumo de alimentos ricos em fibras (grupo I), alimentos ricos em carboidratos simples (grupo II) e alimentos ricos em gordura saturada (grupo III), de adultos do Estado de Pernambuco, 2006 de consumo de alimentos protetores e de alimentos-fonte de gorduras saturadas foram verificadas entre os indivíduos não fumantes. O consumo de alimentos-fonte de gorduras saturadas foi mais elevado entre os indivíduos que relataram o consumo de álcool nos 30 dias anteriores à realização da pesquisa (Tabela 2).

Maior consumo de alimentos-fonte de carboidratos simples foi verificado entre os indivíduos com baixo peso. Não foi verificada associação do nível de atividade física e da CC com o consumo de alimentos dos três grupos estudados (Tabela 2).

\section{S C U S S Ã O}

O maior consumo de alimentos-fonte de carboidratos simples, observado na população adulta pernambucana, significativamente maior do que o consumo de frutas, legumes e hortaliças, reflete, em princípio, o processo de transição nutricional pelo qual passa a população de países desenvolvidos e em desenvolvimento. Esse achado vem alertar para o risco de uma dieta inadequada e seus efeitos deletérios à saúde do indivíduo, considerando que o consumo exacerbado de alimentos-fonte de carboidratos simples pode induzir ao acúmulo de ganho de peso corporal e à elevação de triglicérides ${ }^{18,19}$. Dados de pesquisas nacionais para o período de 1975 a 2003 $20-22$ revelam que a população brasileira tem incorporado hábitos alimentares típicos dos países desenvolvidos, isto é, um maior consumo de alimentos industrializados (refrigerantes e embutidos) em detrimento do consumo de produtos regionais e com tradição cultural, como arroz, feijão, farinhas de mandioca e de milho.

Análise de pesquisas realizadas entre as décadas de 1960 e 1980 sobre consumo alimentar em áreas metropolitanas do Brasil aponta que houve uma estagnação ou, até mesmo, redução do consumo de leguminosas, verduras, legumes, frutas e sucos naturais ${ }^{5}$. Essas mudanças observadas no padrão alimentar repercutem em inges- 
Tabela 2. Medianas e intervalos interquartílicos dos escores de consumo alimentar segundo variáveis socioeconômicas, demográficas, comportamentais e antropométricas em adultos do Estado de Pernambuco, 2006.

\begin{tabular}{|c|c|c|c|c|c|c|}
\hline \multirow{2}{*}{ Variável } & \multicolumn{6}{|c|}{ Grupo de alimentos $^{\dagger}$} \\
\hline & \multicolumn{2}{|c|}{ I } & \multicolumn{2}{|c|}{$\|$} & \multicolumn{2}{|c|}{ III } \\
\hline Idade (anos) & \multicolumn{2}{|c|}{$n=1571$} & \multicolumn{2}{|c|}{$n=1577$} & \multicolumn{2}{|c|}{$n=1571$} \\
\hline $25 \mid-30$ & 2,3 & $1,7-3,2$ & $4,4^{\mathrm{a}}$ & $3,1-5,4$ & $3,7^{\mathrm{a}}$ & $2,7-4,9$ \\
\hline $30 \mid-40$ & 2,3 & $1,8-3,2$ & $4,2^{\mathrm{a}}$ & $3,1-5,3$ & $3,5^{b}$ & $2,5-4,5$ \\
\hline $40 \mid-50$ & 2,3 & $1,6-3,1$ & $4,1^{\mathrm{a}}$ & $3,1-5,3$ & $3,4^{c}$ & $2,4-4,4$ \\
\hline $50 \mid-60$ & 2,3 & $1,6-3,2$ & $3,8^{\mathbf{b}}$ & $2,6-5,2$ & $2,8^{\mathrm{d}}$ & $2,1-4,0$ \\
\hline$p$-valor ${ }^{*}$ & 0,759 & & 0,051 & & 0,000 & \\
\hline Sexo & \multicolumn{2}{|c|}{$n=1576$} & \multicolumn{2}{|c|}{$n=1575$} & \multicolumn{2}{|c|}{$n=1571$} \\
\hline Masculino & 2,3 & $1,8-3,3$ & 4,2 & $3,0-5,4$ & 3,5 & $2,4-4,5$ \\
\hline Feminino & 2,3 & $1,7-3,1$ & 4,2 & $3,1-5,3$ & 3,5 & $2,5-4,6$ \\
\hline$p$-valor ${ }^{*}$ & 0,329 & & 0,894 & & 0,638 & \\
\hline Escolaridade & \multicolumn{2}{|c|}{$n=1576$} & \multicolumn{2}{|c|}{$n=1575$} & \multicolumn{2}{|c|}{$n=1571$} \\
\hline 이-14 anos & $2,2^{\mathrm{a}}$ & $1,5-3,0$ & $4,0^{\mathrm{a}}$ & $2,7-5,1$ & $3,1^{\mathrm{a}}$ & $2,2-4,2$ \\
\hline $5 \mid-18$ anos & $2,5^{\mathbf{b}}$ & $1,9-3,2$ & $4,5^{b}$ & $3,3-5,5$ & $3,8^{b}$ & $2,9-4,7$ \\
\hline$\geq 9$ anos & $2,6^{c}$ & $2,0-3,6$ & $4,5^{b}$ & $3,4-5,5$ & $4,1^{c}$ & $3,0-5,1$ \\
\hline$p$-valor ${ }^{*}$ & 0,000 & & 0,000 & & 0,000 & \\
\hline Área de residência & \multicolumn{2}{|c|}{$n=1576$} & \multicolumn{2}{|c|}{$n=1577$} & \multicolumn{2}{|c|}{$n=1571$} \\
\hline Região metropolitana do Recife & 2,3 & $1,7-3,1$ & $4,5^{a}$ & $3,4-5,3$ & $3,9^{\mathrm{a}}$ & $3,0-4,7$ \\
\hline Interior urbano & 2,4 & $1,7-3,3$ & $4,3^{\mathrm{a}}$ & $2,9-5,4$ & $3,5^{\mathbf{b}}$ & $2,5-5,0$ \\
\hline Interior rural & 2,3 & $1,7-3,1$ & $4,0^{b}$ & $2,8-5,2$ & $3,2^{c}$ & $2,2-4,3$ \\
\hline$p$-valor ${ }^{*}$ & 0,606 & & 0,000 & & 0,000 & \\
\hline Renda familiar per capita & \multicolumn{2}{|c|}{$n=1556$} & \multicolumn{2}{|c|}{$n=1557$} & \multicolumn{2}{|c|}{$n=1551$} \\
\hline $1^{\circ}$ quartil & $2,1^{\mathrm{a}}$ & $1,3-2,7$ & $3,9^{\mathrm{a}}$ & $2,6-5,0$ & $2,9^{\mathrm{a}}$ & $2,0-4,0$ \\
\hline $2^{\circ}$ quartil & $2,3^{\mathbf{b}}$ & $1,5-3,1$ & $4,0^{\mathbf{b}}$ & $2,8-5,3$ & $3,4^{\mathbf{b}}$ & $2,4-4,5$ \\
\hline $3^{\circ}$ quartil & $2,4^{c}$ & $1,9-3,3$ & $4,4^{c}$ & $3,3-5,4$ & $3,6^{\mathbf{b}}$ & $2,7-4,7$ \\
\hline $4^{\circ}$ quartil & $2,7^{d}$ & $2,0-3,6$ & $4,5^{c}$ & $3,3-5,5$ & $3,9^{c}$ & $2,9-4,9$ \\
\hline$p$-valor ${ }^{*}$ & 0,000 & & 0,000 & & 0,000 & \\
\hline \multicolumn{7}{|l|}{ Tabagismo } \\
\hline Fumante & $2,2^{\mathrm{a}}$ & $1,5-3,0$ & 4,1 & $2,8-5,3$ & $3,5^{\mathrm{a}}$ & $2,4-4,5$ \\
\hline Não fumante & $2,4^{\mathbf{b}}$ & $1,7-3,3$ & 4,3 & $3,1-5,3$ & $3,6^{\mathrm{ab}}$ & $2,5-4,6$ \\
\hline Ex-fumante & $2,3^{\mathrm{ab}}$ & $1,8-3,1$ & 4,1 & $3,1-5,3$ & $3,3^{\mathrm{ac}}$ & $2,3-4,2$ \\
\hline$p$-valor ${ }^{*}$ & 0,026 & & 0,473 & & 0,055 & \\
\hline Consumo de álcool & & & & & & \\
\hline Não & 2,4 & $1,7-3,1$ & 4,1 & $3,0-5,3$ & 3,3 & $2,4-4,4$ \\
\hline $\operatorname{sim}$ & 2,3 & $1,7-3,3$ & 4,3 & $3,0-5,3$ & 3,8 & $2,7-4,7$ \\
\hline$p$-valor ${ }^{*}$ & 0,483 & & 0,591 & & 0,001 & \\
\hline Atividade física ${ }^{\ddagger}$ & & & & & & \\
\hline Insuficientemente ativos & 2,4 & $1,8-3,2$ & 4,2 & $3,0-5,3$ & 3,4 & $2,5-4,4$ \\
\hline Suficientemente ativos & 2,3 & $1,7-3,2$ & 4,2 & $3,0-5,3$ & 3,5 & $2,4-4,6$ \\
\hline$p$-valor ${ }^{*}$ & 0,333 & & 0,776 & & 0,854 & \\
\hline Estado nutriciona $\$^{\S}$ & & & & & & \\
\hline Baixo Peso $($ IMC $<18,5 \mathrm{kgm} / 2)$ & 2,2 & $1,7-2,8$ & $5,1^{\mathrm{a}}$ & $3,9-6,3$ & 3,7 & $2,5-5,3$ \\
\hline Eutrofia (IMC $\geq 18,5 \mathrm{~kg} / \mathrm{m}^{2}$ e $<25 \mathrm{~kg} / \mathrm{m}^{2}$ ) & 2,4 & $1,6-3,2$ & $4,3^{\mathbf{b}}$ & $3,0-5,3$ & 3,5 & $2,4-4,5$ \\
\hline Excesso de Peso $\left(I M C \geq 25 \mathrm{~kg} / \mathrm{m}^{2}\right)$ & 2,3 & $1,7-3,2$ & $4,1 b^{c}$ & $3,0-5,3$ & 3,5 & $2,5-4,5$ \\
\hline$p$-valor ${ }^{\star}$ & 0,567 & & 0,024 & & 0,716 & \\
\hline Circunferência da cintura & & & & & & \\
\hline Normal & 2,3 & $1,7-3,2$ & 4,3 & $3,1-5,4$ & 3,5 & $2,5-4,5$ \\
\hline Elevada" & 2,3 & $1,7-3,1$ & 4,1 & $3,0-4,3$ & 3,5 & $2,5-4,6$ \\
\hline$p$-valor ${ }^{\star}$ & 0,994 & & 0,086 & & 0,707 & \\
\hline
\end{tabular}

*Teste de Krukal Wallis. Teste a posteriori: Teste "U" de Mann Whitney. "Grupo I: frutas, legumes, hortaliças e leguminosas. Grupo II: bolo, biscoito, açúcar e refrigerante. Grupo III: carnes com gordura, frango com pele, vísceras, embutidos, leite e derivados, gorduras e frituras; " ${ }^{\text {Suficientemente }}$ ativos: atividades moderada, vigorosa ou caminhada por tempo $\geq 150$ minutos/semana. Insuficientemente ativos: indivíduos com atividades moderada, vigorosa ou caminhada <150min/semana; ${ }^{\S} \mathrm{OMS}, 1998$; ${ }^{\mathrm{I}} \mathrm{CC}$ elevada: $>80 \mathrm{~cm}$ para mulheres e >94cm para homens; ${ }^{\text {a,b,c,d }}$ Letras diferentes significam diferenças estatísticas entre as categorias. 
tão elevada de lipídeos e de carboidratos simples, que, associada ao insuficiente consumo de frutas e de hortaliças e ao crescente sedentarismo, traz como consequência o aumento na prevalência do excesso de peso, das DCV e de outras Doenças Crônicas Não-Transmissíveis (DCNT) ${ }^{21}$.

A alimentação contribui de várias formas para a determinação do risco cardiovascular e sua composição pode constituir fator de risco ou de proteção ${ }^{23}$. As gorduras saturada e trans são reconhecidamente os principais componentes de elevação da concentração plasmática de colesterol e das lipoproteínas de baixa densidade. O baixo consumo de frutas, legumes e verduras, por sua vez, encontra-se entre os cinco principais fatores de risco associados à ocorrência de DCNT ${ }^{24}$.

O perfil alimentar de uma população é consequência de uma complexa interação de características multidimensionais que incluem fatores ambientais, comportamentais, demográficos, socioeconômicos e culturais ${ }^{25}$.

O maior consumo de frutas, legumes e hortaliças, observado nos indivíduos de maior renda e escolaridade, corrobora os achados na literatura, que evidenciam frequentemente a associação entre maior nível socioeconômico e cultural e consumo desses alimentos ${ }^{10,11,22}$. Entretanto, a constatação de que renda mais alta e escolaridade também se associaram ao consumo de alimentos-fonte de carboidratos simples e gorduras saturadas nos permite inferir que o nível socioeconômico influencia o consumo, mas não determina a qualidade da dieta. Sendo assim, os adultos pernambucanos de maior renda e escolaridade, que, em princípio, estariam mais protegidos de DCNT em virtude do maior consumo de alimentos protetores, não se beneficiariam desse hábito, uma vez que apresentaram maior consumo de alimentos-fonte de carboidratos simples e gorduras saturadas. Fórnes et al. ${ }^{14}$, ao estudarem a associação entre os escores alimentares e os níveis lipêmicos em adultos de São Paulo, identificaram associação direta entre o consumo de alimentos- -fonte de gorduras saturadas e os níveis de Colesterol Total (CT) e Lipoproteína de Baixa Densidade-colesterol (LDL-c) e uma associação inversa entre o consumo de alimentos protetores (fontes de fibras) e os níveis de CT e LDL-c. Damon \& Drewnowski ${ }^{26}$, buscando explicar os mecanismos causais da associação entre o nível socioeconômico e a qualidade da dieta, concluíram que muito além do conhecimento nutricional ou motivação para o consumo, o custo dos alimentos seria um aspecto limitante para a determinação de sua aquisição.

A similaridade no consumo dos três grupos de alimentos observada entre os sexos é um resultado inesperado, considerando que as mulheres parecem reconhecer e valorizar mais a relação entre alimentação e saúde, além de culturalmente serem responsabilizadas pelo preparo das refeições ${ }^{27}$. Além disso, diversos estudos nacionais ${ }^{10-12,22}$ apontam um maior consumo de frutas, legumes e hortaliças pelo sexo feminino, o que reforçaria a concepção de que as mulheres teriam uma maior preocupação com sua alimentação. A ausência de associação entre consumo de alimentos-fonte de carboidratos simples e gorduras saturadas e sexo diverge de dados de inquérito telefônico realizado nas capitais do país, que apontaram maior consumo de carnes com excesso de gordura entre os homens ${ }^{13}$.

A homogeneidade no consumo de frutas, legumes e hortaliças segundo a distribuição etária contraria os resultados verificados em outros estudos $^{10-12,22,28}$ que evidenciam maior consumo desse grupo de alimentos por indivíduos de maior idade. Quanto ao consumo de alimentos-fonte de carboidratos simples e gorduras saturadas, o menor consumo observado nos indivíduos mais velhos ( $50 \vdash 60$ anos) poderia ser atribuído a um possível efeito coorte. Estudo nacional com amostra representativa da população brasileira, avaliando associação entre idade e consumo de frutas e hortaliças, encontrou maior consumo desses alimentos entre os indivíduos de maior idade, atribuindo ao fator "idade" esse efeito coorte, pois indivíduos mais velhos estiveram menos 
expostos à alimentação moderna, que inclui alimentos processados de elevada densidade energética ${ }^{28}$.

O menor consumo de alimentos-fonte de gorduras saturadas e carboidratos simples verificado entre os indivíduos residentes do IR é um resultado esperado, uma vez que por se tratar de uma área rural, supõe-se que essa população tenha um menor acesso aos alimentos industrializados. Entretanto, em outros países em desenvolvimento, a exemplo do México ${ }^{29}$, as comunidades rurais já começam a seguir o padrão de dieta similar ao das populações urbanas, com aumento no aporte energético proveniente de alimentos de origem animal e industrializados (ricos em gorduras, açúcares e sal e pobres em fibras), com consequente aumento no consumo de colesterol e gorduras saturadas. No entanto, essa tendência não foi observada entre os adultos pernambucanos, considerando que o consumo dos grupos de alimentos de risco foi superior entre a população da Região Metropolitana de Recife.

O maior consumo de frutas, legumes e hortaliças em adultos pernambucanos não fumantes é um comportamento que corresponde aos resultados descritos por outros autores ${ }^{11,12}$ que sugerem a existência de uma aglutinação de comportamentos que caracterizam um estilo de vida saudável e, em contrapartida, identificam também uma aglutinação dos comportamentos considerados de risco. Entretanto, essa suposição não pode ser inferida neste estudo, uma vez que os adultos não fumantes também apresentaram maior consumo de alimentos-fonte de gorduras saturadas.

É possível encontrar na literatura diferentes resultados acerca da associação entre o consumo de frutas, legumes e hortaliças e o nível de atividade física. A ausência de associação observada na nossa casuística corrobora os achados descritos por Mondini et al. ${ }^{11}$, que, ao analisarem o consumo de frutas e hortaliças por adultos de Ribeirão Preto (SP), encontraram consumo semelhante em indivíduos pouco, moderadamente e muito ati$\operatorname{vos}^{11}$. No entanto, outros autores constataram que indivíduos com maior nível de atividade física apresentaram maior consumo de frutas, legumes e verduras ${ }^{10,12}$.

O maior consumo de alimentos-fonte de gorduras saturadas observado entre os indivíduos que relataram o consumo de álcool pode ser atribuído ao fato de os alimentos comumente associados à ingestão de bebidas alcoólicas serem ricos em gorduras. É importante destacar que a presença simultânea da exposição ao consumo do álcool e ao elevado consumo de gorduras saturadas pode potencializar os efeitos deletérios desses marcadores de risco no estado nutricional e na saúde do indivíduo.

Sabe-se que o desenho transversal, utilizado neste estudo, não é o delineamento mais apropriado para investigar a associação entre consumo alimentar e características antropométricas, especialmente, pela potencial causalidade reversa nessa relação. Portanto, a associação entre o baixo peso e o maior consumo de alimentos-fonte de carboidratos simples poderia ser interpretada sob esse viés. Nesse caso, os indivíduos com baixo peso aumentariam o consumo desses alimentos para incrementar o aporte energético rico da dieta e promover o ganho ponderal. A associação de padrões alimentares com o excesso de peso tem sido objeto de interesse em outros estudos, entretanto são ainda escassas as investigações que avaliam a associação específica entre baixo peso e consumo de alimentos.

Admite-se, naturalmente, a existência de limitações metodológicas neste estudo, como, por exemplo, o fato de o modelo conceitual dentre os seus determinantes hipotéticos causais não ter incluído os aspectos culturais, como as crenças, comportamentos e tabus alimentares, que, em tese, devem ter um peso significativo na determinação dos hábitos alimentares. De certa forma, o modelo escolhido para explicação do desfecho estudado foi reducionista, partindo-se do pressuposto de que fatores ligados aos aspectos culturais têm importante interferência tanto sobre o registro das informações de consumo alimentar quanto sobre os hábitos dietéticos praticados. 
Os escores alimentares, proposta metodológica utilizada nessa investigação, refletem a qualidade de dieta ${ }^{14}$, mas ainda não foi arbitrado um ponto de corte para categorizar em "adequado" ou "inadequado" o consumo de cada grupo de alimentos. Estudos que determinem os pontos de discriminação diagnóstica para categorizar os escores numa perspectiva de associação com a ocorrência ou proteção dessas doenças ampliariam a utilização dessa metodologia na avaliação de padrões alimentares de grupos populacionais, bem como permitiriam a utilização de modelos de análise multivariada para estudar a associação entre o desfecho e as variáveis potencialmente preditoras. Em termos de lógica formal, é um avanço, mas é necessário maior acúmulo de experiências e análises para se apreciar sua validade.

\section{O N CLUS Ã O}

É possível evidenciar que o padrão de consumo, com predomínio de carboidratos simples em detrimento do consumo de frutas, legumes e hortaliças, seria o reflexo de uma sociedade moderna e do processo de transição nutricional, que sofre influência de fatores socioeconômicos, demográficos e comportamentais. Entretanto, dificilmente variáveis associadas exclusivamente ao indivíduo permitiriam explicar a complexidade do consumo de uma população. É importante destacar que as características qualitativas da dieta são importantes na definição do estado de saúde de uma população, principalmente no que diz respeito às doenças crônicas degenerativas da idade adulta.

O presente estudo fornece subsídios importantes para o estado de Pernambuco e para a Região Nordeste do País, possibilitando que as informações obtidas sejam monitoradas a fim de serem identificadas tendências no comportamento alimentar dessa população.

Mudança de hábito e de comportamento requer esforço coletivo, como políticas de saúde mais abrangentes que objetivem a valorização de consumo de alimentos mais saudáveis. A consolidação de iniciativas governamentais é fundamental para reverter a atual situação de morbimortalidade das doenças não-transmissíveis, dentre as quais as doenças cardiovasculares, que ocupam as primeiras posições de causalidade de mortes.

\section{REFERÊNCIAS}

1. Lopes ACS, Caiaffa WT, Sichieri R, Mingoti AS, Lima-Costa MF. Consumo de nutrientes em adultos e idosos em estudo de base populacional: Projeto Bambuí. Cad Saúde Pública. 2005; 21(4):1201-9.

2. Petribu MMV, Cabral PC, Arruda IKG. Estado nutricional, consumo alimentar e risco cardiovascular: um estudo em universitários. Rev Nutr. 2009; 22(6):837-46. doi: 10.1590/\$1415-52732009000 600005.

3. Coyle D, Buxton MJ, O'Brien BJ. Measures of importance for economic analysis based on decision modeling. J Clin Epidemiol. 2003; 56(10):989-97.

4. Mondini L, Monteiro CA. Mudanças no padrão de alimentação na população urbana brasileira (19621988). Rev Saúde Pública. 1994; 28(6):433-9.

5. Monteiro CA, Mondini L, Costa RBL. Mudanças na composição e adequação nutricional da dieta familiar nas áreas metropolitanas do Brasil (19881996). Rev Saúde Publica. 2000; 34(3):251-8.

6. Machado FMS, Simões NA. Análise custo-efetividade e índice de qualidade da refeição aplicados à estratégia global da OMS. Rev Saúde Pública. 2008; 42(1):64-72.

7. Costa ACV, Priore SE, Sabarense CM, Franceschini SCC. Questionário de freqüência de consumo alimentar e recordatório de 24 horas: aspectos metodológicos para avaliação da ingestão de lipídeos. Rev Nutr. 2006; 19(5):63-41. doi: 10.1590/S 1415-52732006000500011.

8. Guedes DP, Guedes JERP. Physical activity, cardiorespiratory fitness, dietary content, and risk factor that cause a predisposition towards cardiovascular disease. Arq Bras Cardiol. 2001; 77(3):251-7.

9. Castro LCV, Franceschini SCC, Priore SE, Pelúzio MCG. Nutrição e doenças cardiovasculares: os marcadores de risco em adultos. Rev Nutr. 2004; 17(3):369-77. doi: 10.1590/\$1415-527320040 00300010

10. Figueiredo ICR, Jaime PC, Monteiro CA. Fatores associados ao consumo de frutas, legumes e 
verduras em adultos da cidade de São Paulo. Rev Saúde Pública. 2008; 42(5):777-85.

11. Mondini L, Moraes AS, Freitas ICM, Gimeno SGA. Consumo de frutas e hortaliças por adultos em Ribeirão Preto, SP. Rev Saúde Pública. 2010; 44(4): 686-94.

12. Campos VC, Bastos JL, Gauche H, Boing AF, Assis MAA. Fatores associados ao consumo adequado de frutas, legumes e verduras em adultos de Florianópolis. Rev Bras Epidemiol. 2010; 13(2): 352-62.

13. Moura EC, Morais Neto OL, Malta DC, Moura L, Silva LN, Bernal R, et al. Vigilância de fatores de risco para doenças crônicas por inquérito telefônico nas capitais do 26 estados brasileiros e no Distrito Federal (2006). Rev Bras Epidemiol. 2008; 11(1): 20-37.

14. Fornés NS, Martins IS, Velasquez-Melendez G, Latorre MRDO. Escores de consume alimentar e níveis lipêmicos em população de São Paulo, Brasil. Rev Saúde Pública. 2002; 36(1):12-8.

15. International Physical Activity Questionnaire [Internet]. 2001 [cited: 2010 Sep. 10]. Available from: <http://www.ipaq.ki.se/ipaq.htm>.

16. Barros MV, Nahas MV. Comportamentos de risco, auto-avaliação no nível de saúde e percepção de estresse entre trabalhadores da indústria. Rev Saúde Pública. 2001; 35(6):554-63.

17. World Health Organization. Obesity: preventing and managing the global epidemic. Geneva: WHO; 1998. Report of a WHO Consultation on Obesity.

18. Bevilacqua MR, Gimento SGA, Matsumura LK, Ferreira SRG, Japanese Brazilians Diabetes Study Group. Hiperlipidemias e fatores dietéticos: estudo transversal entre nipo-brasileiros. Arq Bras Endocrinol Metab. 2007; 51(4):547-58.

19. Santos ALT, Weiss T, Duarte CK, Azevedo MJ, Zelmanovitz T. Análise crítica das recomendações da Associação Americana de Diabetes para doença cardiovascular no diabetes melito. Arq Bras Endocrinol Metab. 2009; 53(5):657-66.
20. Instituto Brasileiro de Geografia e Estatística. Estudo nacional da despesa familiar: consumo alimentar - antropometria. Rio de Janeiro: IBGE; 1977. v.1.

21. Instituto Nacional de Alimentação e Nutrição. Pesquisa Nacional sobre Saúde e Nutrição: PNSN-1989 - arquivo de dados da pesquisa. Brasília: INAN; 1990.

22. Instituto Brasileiro de Geografia e Estatística. Pesquisa de orçamentos familiares 2002-2003: análise da disponibilidade domiciliar de alimentos e do estado nutricional no Brasil. Rio de Janeiro: IBGE; 2004.

23. Neumann Al, Shirassu MM, Fisberg RM. Consumo de alimentos de risco e proteção para doenças cardiovasculares entre funcionários públicos. Rev Nutr. 2006; 19(1):19-28. doi: 10.1590/\$1415-52 732006000100002 .

24. World Health Organization. The World Health Report 2002: reducing risks, promoting healthy life. Geneva: WHO; 2002.

25. Whichelow MJ, Prevost AT. Dietary patterns and their associations with demographic, lifestyle and health variables in a random sample of British adults. Br J Nutr. 1996; 76(1):17-30

26. Darmon N, Drewnowski A. Does social class predict diet quality? Am J Clin Nutr. 2008; 87(5):1107-17.

27. Baker $\mathrm{AH}$, Wardle J. Sex differences in fruit and vegetable intake in older adults. Appetite. 2003; 40(3):269-75.

28. Jaime PC, Machado FMS, Westphal MF, Monteiro CA. Nutritional education and fruit and vegetable intake: a randomized community trial. Rev Saúde Pública. 2007; 41(1):1-4.

29. Arenas JA, Pérez ME, Villasana AC. Evaluación de los patrones alimentarios y la nutrición em cuatro comunidades rurales. Salud Pública Méx. 1998; 40(5):398-407.
Recebido em: 8/11/2011 Aprovado em: 16/5/2012 
